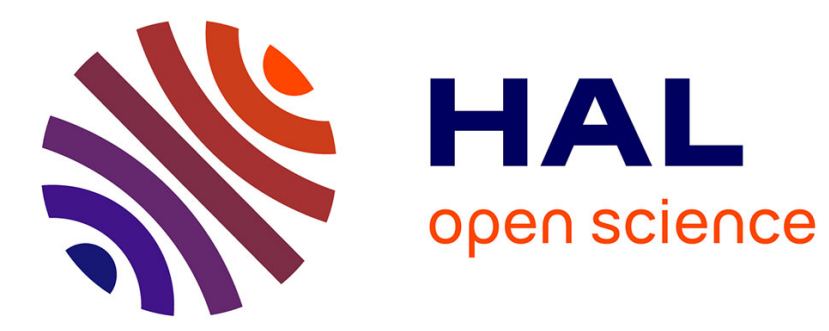

\title{
Approche des relations entre eau et agriculture à AgroParisTech
}

Philippe Martin, Jean-Stéphane Bailly, Flavie Cernesson

\section{To cite this version:}

Philippe Martin, Jean-Stéphane Bailly, Flavie Cernesson. Approche des relations entre eau et agriculture à AgroParisTech. Pour : enquêtes et témoignages, 2012, 2013 (213), pp.203-214. hal-01526064

\section{HAL Id: hal-01526064 \\ https://hal.science/hal-01526064}

Submitted on 19 Nov 2019

HAL is a multi-disciplinary open access archive for the deposit and dissemination of scientific research documents, whether they are published or not. The documents may come from teaching and research institutions in France or abroad, or from public or private research centers.
L'archive ouverte pluridisciplinaire HAL, est destinée au dépôt et à la diffusion de documents scientifiques de niveau recherche, publiés ou non, émanant des établissements d'enseignement et de recherche français ou étrangers, des laboratoires publics ou privés. 


\section{Philippe MARTIN 1}

Professeur d'Agronomie, UFR DEFI ; UMR SAD APT

Jean Stéphane BAILLY $^{2}$

ICPEF, UFR IGTE; UMR TETIS-LISAH

\section{Flavie CERNESSON ${ }^{3}$}

Maître de conférences en Hydrologie, UFR SIED; UMR TETIS AgroParisTech,

\section{Approche des relations entre eau et agriculture à AgroParisTech}

Les relations entre eau et agriculture mettent en œuvre une diversité de compétences qui nécessitent un apprentis- sage dès les bancs de l'école. Cet acquis initial sera enrichi par les expériences de terrain qui feront elles-mêmes naître de nouveaux besoins de formation. Ces besoins peuvent être de l'ordre de l'expertise à affiner sur un sujet particulier, ou de l'ordre de compétences d'animation

\footnotetext{
1 UFR DEFI : Unité de Formation Recherche Décision Exploitations Filières ; UMR SAD APT: Unité mixte de Recherche Sciences pour l'Action et le Développement Activités Produits Territoires [AgroParisTech, INRA). 2 IGTE : Information Géographique Territoire Environnement ; TETIS Territoire, Environnement Télédétection et Information Spatiale [AgroParisTech, IRSTEA lex CEMAGREFJ, CIRAD). LISAH Laboratoire d'interactions sol-agrosystèmes-hydrosystèmes. 3 SIED : Science et Ingénierie de l'Eau et des Déchets; TETIS Territoire, Environnement Télédétection et Information Spatiale (AgroParisTech, IRSTEA (ex CEMAGREFI. CIRADJ.

4 SIAFEE : Sciences et Ingénierie Agronomiques, Forestières de l'Eau et de [Environnement.
} 
d'équipes travaillant de façon pluridisciplinaire sur le sujet. Iobjet de cet article est de présenter comment les relations eau-agriculture sont abordées au sein d'une école comme AgroParisTech.

\section{AgroParisTech, une école originale issue de trois héritages}

AgroParisTech, de son vrai nom Institut des sciences et industries du vivant et de l'environnement, résulte de la fusion en 2007 de trois écoles avec leur culture et leur corpus disciplinaire : I'INA P-G, I'ENSIA et I'ENGREF. LINA P-G avait une vocation pluridisciplinaire large et formait avant tout des ingénieurs et des masters travaillant sur les productions agricoles animales et végétales, leur transformation et leur valorisation, tout en traitant des impacts sur l'environnement et la société. LENGREF avait une double mission : celle de former les cadres du corps du GREF et celle de former les ingénieurs forestiers avec la FIF basée à Nancy. Les différentes voies de formation des ingénieurs du GREF (fonctionnaires et civils] permettaient aussi d'accueillir des étudiants extérieurs à ce cursus à des niveaux post-master. LENSIA quant à elle avait une reconnaissance forte dans le domaine de la transformation des productions agricoles au sein du secteur agroalimentaire.

La création d'AgroParisTech, l'évolution du corps des IGREF vers celui des IPEF et la profonde réforme des masters se sont traduites par des évolutions importantes à rappeler pour bien comprendre la place que prennent les relations eau-agriculture dans ce dispositif. La nouvelle structure comporte un cursus ingénieur (trois années d'école avec un stage de fin d'étude de six moisi, ainsi qu'un cursus master plutôt orienté recherche Imaster 1 puis master 2 avec un stage de recherche de cinq mois destiné à des étudiants souhaitant ensuite majoritairement continuer en thèse, notamment au sein de l'école doctorale ABIES portée par AgroParisTech. Ces deux voies de formation initiale sont complétées par des formations executive, pcst-rnaster non doctorales et formations qualifiantes ou certifiantes (ex-formation continue] pour le management. la gestion et l'administration du vivant et de l'environnement. On peut signaler à ce niveau des formations diplômantes de mastères spécialisés destinées à des diplômés de l'enseignement supérieur qui souhaitent évoluer vers des fonctions de management. On trouve aussi une large gamme de sessions de formations qualifiantes proposées aux professionnels des secteurs couverts par l'établissement. Depuis la rentrée 2011. il y a une disjonction forte entre la formation d'IPEF (réduite à un an avec un programme commun pour tous les étudiants, coconstruit entre l'école des Ponts ParisTech et AgroParisTechl et les formations de mastères spécialisés.

Dans les lignes qui suivent nous présenterons les formations traitant des liens entre l'eau et l'agriculture en distinguant les cursus ingénieur, master let poursuite en doctorat]. et executive. Notre propos n'est pas de faire un catalogue exhaustif de ce qui peut se faire à chacun de ces niveaux, mais plutôt de signaler des types de connaissances et savoir-faire importants dispensés en fonction des besoins identifiés à chaque niveau.

\section{Le cursus ingénieur}

\section{Rappel du ruban pédagogique}

Les étudiants sont majoritairement recrutés après deux années de classe préparatoire. Larrivée en école d'ingénieur va se traduire par la mobilisation de nouvelles disciplines : agronomie, sciences animales, foresterie mais aussi économie, gestion. sociologie ou droit de l'environnement pour n'en citer que quelques-unes. Elle se traduit aussi par une articulation progressive de ces différentes disciplines entre elles. En première année, les 340 étudiants, qui avant la fusion des écoles étaient recrutés dans trois cursus différents (agronome, agro-alimentaire, forestier]. suivent depuis la rentrée 2010 un cursus commun. À l'issue de cette première année, ils doivent s'inscrire pour leur deuxième année dans l'un des quatre domaines proposés par l'école:

: Productions, filières, territoires pour le développement durable

2 : Ingénierie des aliments. biomolécules et énergie :

3 : Gestion et ingénierie de l'environnement

4: Ingénierie et santé: homme. bioproduits. environnement.

La notion de domaine correspond à une première orientation qui se traduit par un cursus spécifique de huit semaines en cours de 2e année. La 3e année correspond à une année de spécialisation qui se termine par un stage de fin d'études de six mois. Elle se fait majoritairement au sein d'un des trois sites de l'établissement [Paris, Massy, Nancy), mais des accords de double diplôme existent avec d'autres écoles françaises et étrangères.

\section{La thématique Eau et agriculture dans le cursus ingénieur}

En tout début de première année, les étudiants sont confrontés aux grands enjeux sociétaux qui constitueront la base de leurs futurs métiers. Leau est l'un des thèmes mis en avant selon quatre angles de vue dont deux concernent directement notre thématique : "L'eau et les agricultures, inter-relations et interdépendances », " La gouvernance de ieeu - quels grands écarts ?». Ces différents angles de vue permettent de présenter l'importance des questions d'usage, de cycle, de gestion de 'eau et de risques liés à l'eau. D'un point de vue pédagogique, ils permettent aussi de problématiser l'enseignement plus académique qui suit cette courte séquence introductive d'une dizaine d'heures.

Par la suite. en première année, les relations eau-agriculture sont 
abordées avant tout du point de vue des mécanismes biophysiques vus à différentes échelles (de la plante à la planète pour le cycle de l'eau). L.étude des mécanismes de base d'échanges d'énergie et de gaz \{vapeur d'eau et dioxyde de carbone) entre écosystèmes et atmosphère permet de comprendre le couplage entre biosphère et climat, et d'aborder les régulations de ces échanges par les écosystèmes. L.analyse des relations entre sol. plante et atmosphère permet de traiter du bilan hydrique pour différentes occupations du sol, agricoles et forestières, en fonction des conditions de milieu (sol, climat local] et de discuter ainsi de systèmes de culture plus ou moins économes en eau. La circulation de l'eau permet aussi d'aborder la thématique de la pollution diffuse en profondeur par les nitrates, ainsi que des problèmes de ruissellement et d'érosion pouvant générer des coulées boueuses dans les agglomérations situées en aval. Ces enseignements permettent dès la première année de mettre en évidence les dimensions temporelles et spatiales des mécanismes et des décisions d'acteurs concernés. La dimension temporelle des successions de culture et le rôle de l'organisation spatiale des systèmes de culture sont ainsi mis en avant. La première année insiste beaucoup sur le niveau de décision de l'exploitation agricole dont on montre les cohérences techniques pour expliquer un certain nombre de pratiques (approche globale de l'exploitation agricole). On donne aussi quelques clés de compréhension de dispositifs multi-acteurs d'ordre réglementaire (ex. de la directive nitrate) en les croisant avec des logiques économiques. Partant de l'exemple des productions animales, notamment en Bretagne, on discute ainsi des marges de manoeuvre des différents acteurs concernés dans un contexte de mondialisation pouvant se traduire par des délocalisations brutales de grands pans de la production animale.

Àce stade de la formation, on reste sur des formats pédagogiques classiques [cours + travaux dirigés). Des espaces de liberté sont toutefois laissés aux étudiants pour leur conférer plus d'autonomie dans leur approche. Les étudiants doivent ainsi réaliser un projet, en petit groupe de quatre à huit étudiants, sur un thème de leur choix validé par un enseignant qui les accompagne dans leur réflexion. Ces projets correspondent à une semaine sur le terrain (en l'absence des enseignants) et font l'objet d'une soutenance orale et d'une remise de rapport écrit qui permettent d'aider les étudiants à aller plus loin dans leur réflexion.

En première année, les étudiants doivent réaliser un stage de quatre semaines. très majoritairement en exploitation agricole. C'est l'occasion pour l'étudiant de confronter les enseignements reçus à AgroParisTech à une certaine réalité de terrain. Réalisé de manière individuelle. ce stage donne lieu à une soutenance orale à partir d'un poster devant des enseignants de différents domaines. La mise en avant de la thématique eau et agriculture dépend beaucoup des contextes et des points de vue des maîtres de stage que les étudiants adoptent quasi systématiquement. La soute- nance orale est l'occasion d'aider les étudiants à prendre du recul par rapport à ce qu'ils ont pu vivre pendant ces quatre semaines.

C'est en fin d'année qu'une séquence d'enseignement. dite Module intégratif, perme aux étudiants d'approfondir de manière encadrée une thématique pluridisciplinaire liée au terrain. Le module intégratif constitue la première expérience de mise en œuvre des connaissances et méthodes apportées par la formation dans différentes disciplines. C'est une étape très appréciée des étudiants qui permet de les préparer pour les exercices plus exigeants de $2 \mathrm{e}$ et $3 \mathrm{e}$ années. Plusieurs modules portent sur La thématique eau et agriculture. I.un d'eux aborde la qualité de l'eau au niveau d'un petit bassin versant Icelui de Grignon) en cherchant à en comprendre les déterminants liés à l'occupation du sol et à la circulation dans le sol et les couches géologiques. Un autre s'intéresse aux outils et méthodes disponibles pour gérer au mieux l'eau dans le champ cultivé, que ce soit du point de vue de l'irrigation ou du drainage. Un troisième module. porté par des enseignants de science politique, vise à initier les élèves aux concepts et méthodes de la gestion patrimoniale des ressources et des milieux Idont notamment ceux qui sont liés à l'eaui. Grâce à un voyage de découverte sur un bassin versant doté d'une institution innovante (Sèvre Nantaise], il permet également de montrer comment les acteurs très différents d'un bassin versant peuvent élaborer un dessein commun et trouver les voies et moyens d'une gestion en patrimoine commun de la ressource eau et des milieux associés. En 2e année, l'enseignement permet des approfondissements à différents niveaux. En tronc commun, les étudiants s'initient aux bases du droit del'environnement dans lequel les relations eau-agriculture occupent une place importante. Les relations eau-agriculture sont peu abordées en enseignement de domaine du fait d'une disjonction un peu artificielle entre production (01 let environnement (03). La rencontre entre les deux approches Agriculture et Eau se fait lors des séquences optionnelles proposées en $2 \mathrm{e}$ année, et notamment dans des modules de longue durée (six semaines) appelés Projet de $2 \mathrm{e}$ année. Ces modules sont dans le prolongement pédagogique du Projet de 1e année présenté plus haut. Pour la séquence projet de $2 \mathrm{e}$ année, on propose aux étudiants une quinzaine de thématiques dont trois entrent directement dans celle de cet article : "Gestion des eaux dans un bassin versant ", "Littoral: interface terre-rner " et "Gestion patrimoniale des territoires». Ces trois modules ont en commun de mobiliser des équipes pluridisciplinaires pour répondre aux questions de partenaires de terrain. Ils associent des phases de terrain d'assez longue durée (deux à trois semaines) à des phases de maturation de la problématique et de traitement des données, le tout avec un fort appui des enseignants. Cette expérience du projet de $2 \mathrm{e}$ année marque souvent très fortement et très positivement les étudiants. Ces trois projets se distinguent par l'angle d'attaque. Pour le projet Gestion des eaux dans 
un bassin versant, l'objectif est de travailler sur le bassin versant d'une petite rivière [de l'ordre de 15 à $20 \mathrm{~km}$ de long, correspondant à une vingtaine de communes) pour répondre à une gamme de questions que se posent les acteurs locaux. Sont particulièrement mises en avant la maîtrise des systèmes d'information géographique (SIG) et les bases de l'hydrologie. Chaque année, un des thèmes retenus porte sur les relations entre eau et agriculture. Pour le projet Littoral (interface terre-merl. I'originalité est d'associer qualité des eaux douces et qualité des eaux de mer aux différentes activités des populations concernées. Alors que les deux précédents projets étaient plutôt portés par des enseignants de sciences biotechniques, le projet "gestion patrimoniale des territoires » est porté par des enseignants de sciences politiques. II a pour projet pédagogique de former les étudiants à une approche stratégique des questions complexes posées par le vivant aux acteurs d'un territoire, sur des problématiques pas nécessairement Limitées à l'eau. Dans cette approche. l'explicitation des attentes des différents acteurs par rapport aux ressources du vivant joue un rôle majeur.

La thématique eau et agriculture apparaît aussi dans des modules de plus courte durée. C'est notamment le cas du module "Gestion de l'eau - problématiques régionales et planétaires», d'une semaine, proposé dans le dispositif Athens, c'està-dire ouvert à un large public composé d'étudiants des grandes écoles parisiennes participant au dispositif. L.enseignement cherche à délivrer les éléments de connaissance utiles pour analyser les situations actuelles, pour prévoir, compte tenu des pressions anthropiques, le sens des évolutions probables et finalement proposer des modes de gestion plus durables. De courte durée et destiné à un public hétérogène, il ne comporte que des cours.

En troisième année du cursus ingénieur, les étudiants choisissent des dominantes d'approfondissement (DA) portées par des collectifs d'enseignants. Les relations eau-agriculture sont particulièrement abordées dans une de ces dominantes : Idea (Ingénierie de l'environnement eaux déchets et aménagements durables). mais le thème est aussi présent dans d'autres, telles que PISTV (Production et innovation dans les systèmes techniques végétaux) et une nouvelle DA qui démarrera en 20122013, Spes (Science politique écologie et stratégie). Nous présenterons la DA Idea. Le Lecteur intéressé pourra se reporter au site de l'école pour s'informer sur le contenu des autres DA.

La DA Idea a été mise en place à la rentrée 2008-2009. La DA est structurée en trois temps: un tronc commun (septembre à décembre), une option [janvier-février] et un stage (mars à août). Les deux options proposées sont actuellement Eau હel déchets (ESDI et Aménagement et gestion de l'eau et des milieux (Ageml. Les relations eauagriculture sont traitées avant tout en tronc commun et dans l'option Agem. En tronc commun, dans un module introductif. les étudiants abordent la manière dont cer- laines sciences économiques et sociales traitent les thématiques environnementales. Líidée n'est pas de rendre les étudiants directement opérationnels en droit ou en économie de l'environnement, mais de les rendre capables d'interagir avec des spécialistes de ces domaines dans leur vie professionnelle. Dans un deuxième module, les étudiants approfondissent leurs connaissances sur les grands cycles biogéochimiques, sur l'impact des polluants sur les milieux et sur l'analyse de risques. II s'agit là d'un approfondissement par rapport à ce qui a pu être vu en première année, en développant notamment l'appréciation des capacités concrètes de régulation de la qualité d'un milieu via les processus biogéochimiques et les pratiques. Le troisième module vise, d'une part à donner des compléments agronomiques sur les liens entre systèmes de culture et pression sur le mileu, d'autre part à permettre aux étudiants de mobiliser les connaissances acquises dans les deux premiers modules dans le cadre d'un jeu de rôle. Le jeu de rôle se place dans une situation de crise environnementale !coulée de boue liée aux pratques aqricolesl. Les étudiants doivent interpréter les rôles de différents acteurs du territoire lagriculteurs, services déconcentrés de l'État, collectivités, agence de l'eau, syndicat d'eau.... L'.objectif est de permettre aux étudiants : (1 J de remobiliser en situation des éléments du cours ; 2] d'effectuer un auto-apprentissage de connaissances opérationnelles via la bibliographie et la recherche sur Internet pour étayer les arguments qu'ils utiliseront pendant le jeu de rôle ; 31 de se faire une idée de la manière dont ils peuvent intervenir dans une réunion à caractère professionnel.

Dans l'option Agem, la thématique Eau-agriculture est abordée dans le module principal intitulé Gire [Gestion intégrée des ressources en eau] dont l'essentiel se déroule à Montpellier afin de bénéficier des apports des équipes Agro ParisTech de ce site en ce domaine, tant pour les sciences biotechniques que socioéconomiques, et auss pour permettre l'étude de milieux contrastant fortement avec le Bassin parisien. Ce module a pour but d'introduire à la gestion de l'eau, et plus précisément à l'évalua- tion des ressources en eau disponibles, leur état et celui du milieu aquatique, l'iden- tification des besoins en termes de quantité et de qualité d'eau pour les différents usages, la connaissance des services liés à l'utilisation de l'eau. Les relations eau- agriculture sont traitées, par des études de terrain réalisées notamment en parte- nariat avec I'Association syndicale autorisée du canal de Gignac, dans le contexte méditerranéen qui cristallise les questions li] de mutation des territoires (puisque l'agriculture se situe dans un cadre péri-urbain] et d'évolution de la demande en eau, des pratiques associées, de la tarification et [ii] de vulnérabilité de la ressource et des milieux dans un contexte de changement climatique.

En parallèle aux enseignements de tronc commun et d'option, les étudiants doivent mener à bien un projet d'ingénieur. Ce projet est à la fois l'aboutissement de l'apprentissage du projet initié en le et 
2e année. C'est aussi un exercice préparatoire au travail qui devra être conduit pour le stage de fin d'études. Les sujets de projets d'ingénieur et de stages de fin d'études reflètent les préoccupations des commanditaires publics et privés. En 2012, sont notamment très présentes les thématiques liées aux suites du Grenelle de l'environnement avec le dispositif Écophyto 2018, la protection des captages mais aussi des démarches prospectives par rapport à l'évolution des usages de l'eau dans un contexte de changement climatique.

\section{Conclusion sur la sensibilisation aux relations eau-agriculture}

\section{dans le cursus ingénieur}

La pédagogie mise en œuvre vise à donner un fonds commun à tous les étudiants avec le tronc commun de le année. Progressivement, les étudiants affinent leurs choix au travers d'enseiqnernents optionnels \{projets de ie et $2 e$ année) et de choix de domaines de 2e année, pour finir par leur choix de DA de 3e année. Nous avons montré en quoi la DA Idea contribuait à préciser les relations eau-agriculture tout en signalant des points de vue différents développés dans d'autres DA. Le panorama de la formation ingénieur serait toutefois incomplet si on omettait de parler de la césure pratiquée par un nombre important d'étudiants entre la 2e et la 3e année. Cette année hors les murs constitue pour beaucoup d'étudiants [près de $50 \%$ ) l'occasion de confronter leurs aspirations à une réalité de terrain très fréquemment rencontrée dans les pays du Sud où les relations eau-agriculture sont très prégnantes.

\section{Le cursus master et le doctorat}

\section{Rappel du ruban pédagogique}

Le cursus master articule deux années de formation (M1 et M2) fortement axées vers la recherche et l'acquisition ultérieure d'un doctorat. Cette voie intéresse autant des étudiants désireux de s'orienter vers des organismes de recherche que ceux désireux de travailler pour des organismes internationaux recrutant au niveau du doctorat.

\section{La thématique Eau et agriculture dans le cursus master}

Le M1 d'AgroParisTech est structuré en quatre mentions, parmi lesquelles la mention Espaces ressources milieux qui regroupe l'essentiel des enseignements relatifs à la thématique Eau-agriculture. Ces enseignements s'apparentent à une partie de ce qui peut être vu en 1 e année ingénieur sur le sujet. En M2, AgroParisTech propose plusieurs spécialités de master qui, chacune à leur manière, abordent la thématique Eau-agriculture. Parmi ces spécialités, celle intitulée Eau et agriculture est au coeur du sujet de cet article, même si d'autres comme Eau et sociétés, De l'agronomie à l'agro-écologie ou Environnement développement territoires et sociétés traitent aussi le sujet avec chacune leur spécificité et sans que l'eau ou l'agriculture soient nécessairement au cœur de la réflexion proposée aux étudiants.

La spécialité de master Eau et agriculture, basée sur le centre AgroParisTech de Montpellier, est réalisée en partenariat avec Montpellier Supagro et l'université de Montpellier 2. Dans un contexte de changements globaux, cette spécialité de création récente forme des spécialistes de la connaissance et de la gestion des ressources en eau et des milieux par et pour l'agriculture. Elle aborde il les principaux enjeux de connaissance relatifs aux processus hydrologiques et biophysiques et ceux de leurs interactions avec les systèmes de productions agricoles aux échelles de la parcelle agricole, des périmètres irrigués et des bassins versants ruraux, mais aussi iil les approches méthodologiques les plus récentes pour |'observation, la représentation et la modélisation des interactions entre agrosystèmes et hydrosys tèmes. Une spécificité de cette spécialité est son orientation vers les problématiques méditerranéennes et du Sud, avec par exemple quatre UE relatives à L'irrigation et à la gestion des périmètres irrigués, mais aussi de par son insertion dans le collectif de recherche montpelliérain [Agropolis international, Pôle mondial de compétitivité sur l'eau). La spécialité de master Eau et société, bien que moins centrée sur les interactions entre eau et agriculture, s'appuie sensiblement sur le même partenariat que la spécialité précédente. L:objectif de cette formation pluridisciplinaire est de fournir des clés de compréhension et d'identifier les enjeux de connaissance de ce qui est imposé par l'objet physique eau et de ce qui est construit par la société. Elle initie aussi aux outils de gestion des services d'eau ou des périmètres irrigués et aux méthodes d'intervention pour la planification concertée de la ressource. Elle s'adresse aux étudiants intéressés par les problématiques de l'eau analysées avec des outils et méthodes des sciences sociales [économie, droit, sociologie, géographie, sciences de gestion principalement). Au sortir de ces formations de master, Les étudiants ont vocation à poursuivre en doctorat, notamment dans les écoles doctorales cohabilitées par AgroParisTech !Abies, Sibaghel.

La poursuite en thèse sur la thématique "Eau et Agriculture ", est réalisée à AgroParisTech au sein de deux écoles doctorales cohabilitées, Abies sur Paris et Sibaghe sur Montpellier. A l'issue d'un cursus de master recherche ou équivalent, de 2 à 3 doctorants par an inscrits dans l'ensemble Sibaghe-Abies réalisent une thèse sur la thématique eau et agriculture. Ces thèses sont réalisées essentiellement dans les laboratoires sous tutelle d"AgroParisTech : UMR G-Eau pour " gestion de l'eau acteurs usages $\gg$ (irrigation, gestion sociale des ressources en eau), UMR TETIS pour «territoires, environnement, télédétection et information spatiale » lhydrologie spatialisée, spatialisation des flux hydriques), UMR SAD-APT [Sciences pour l'Action et le Développement Action Produits et Territoires) et UMR Agronomie. 


\section{Conclusion sur le master et le doctorat}

Les formations de master, et notamment celles que nous venons d'évoquer, permettent aux étudiants d'évoluer vers un doctorat pour intégrer les instituts de recherche et les organismes internationaux. La formation de master et de doctorat peut aussi être valorisée dans les secteurs publics et privés non directement liés à la recherche [bureaux d'étude et de conseill. La thématique Eau agriculture est particulièrement propice à ce type de trajectoire.

\section{La formation executive dont le post-master diplômant (mastère spécialisé)}

\section{Rappel du ruban pédagogique}

En complément des formations initiales, les formations continuées à destination des professionnels ont pour objectifs de développer des compétences d'assemblage (management, socio-technique). en s'appuyant sur les savoirs et les savoir-faire des apprenants et sur les avancées scientifiques issues de nos expertises et de nos recherches. La formation executive d'AgroParisîech est structurée en quatre gammes de programmes visant à répondre à des demandes différentes :

- la gamme 1 [mastères spécialisés temps pleinl propose une formation diplômante en un an à des professionnels qui interrompent leur carrière pour une formation

- la gamme 2 [mastàres spécialisés en alternance) propose une formation diplốmante en un an à des personnes qui restent en poste dans leur entreprise

- la gamme 3 !certificats d'études avancéesl propose une formation certifiante basée sur un parcours de formation court, où les acquisitions de compétences sont validées par un jury;

- la gamme 4 !forrnations qualifiantes, ex-formation continuel propose des modules courts (deux à cinq jours] à des professionnels qui viennent acquérir des compétences sur un sujet ou sur un domaine restreint. Ces modules peuvent être proposés en catalogue ouvert ou mis au point à la demande sur une commande spécifiée.

Les mastères spécialisés, très adaptatifs au marché de l'emploi, permettent à des cadres d'acquérir une double compétence ou de faciliter une réorientation. Si la poursuite d'études n'est pas une priorité pour ce type de formation, les mastères constituent des voies d'approfondissement intéressantes pour de jeunes professionnels souhaitant travailler dans de grands groupes et si possible à l'international. Ienseignement pour de petits effectifs. le suivi du projet professionnel personnel, la pédagogie par projet et le partenariat fort avec les entreprises constituent les fondamentaux de la pédagogie déployée.

\section{La thématique Eau et agriculture dans le cursus mastère spécialisé}

Depuis 1984, le mastère spécialisé Gestion de l'eau a pour objectif de former des cadres intervenant dans la gestion des ressources en eau et des services associés tels que la protection des ressources, les rivières et les milieux aquatiques, les services d'eau potable et d'assainissement en abordant à la fois les aspects socio-techniques comme les aspects économiques et réglementaires. Iagriculture est présente au travers des usages de l'eau et des pratiques nécessaires (évaluation des ressources, conception, gestion et diagnostic de périmètres irrigués, tarificationl et de son impact sur la ressource et sur les hydrosystèmes. À cette relation stricto sensu, se rajoutent les services environnementaux rendus par l'agriculture, notamment dans l'aménagement de l'espace et la préservation des milieux, ou de la réduction de l'impact des inondations. On considère la gestion de l'eau de façon intégrée et territorialisée: il s'agit donc d'analyser ou d'organiser une gestion de l'eau multi-usages et multi-niveaux. Les cas traités sont situés tant en France que dans les pays méditerranéens et en international. Si l'enseignement se fait maintenant sous la forme classique de conférences et de travaux dirigés dans des modules dédiés Eau et agriculture, Eau et développement, la problématique est aussi présente dans les modules Qualité de l'eau (impact des activités agricoles sur les ressources superficielles et souterraines). Hydraulique à surface libre [fonctionnement des canaux et des ouvrages hydrauliques] et Hydrologie [évaluation des ressources disponiblesl... II est bon de signaler la coopération avec lę Maroc (/AV]. la Tunisie IInatl et I'Algérie, avec l'appui du Cemagref/IRSTEA, qui, pendant plusieurs années, a permis la réalisation de projets d'ingénieurs dédiés à différents publics [étudiants en formation initiale, mastères spécialisés, experts. scientifiques, et professicnnels] dans des opérations intensives de deux semaines sur le terrain.

D'un point de vue institutionnel, AgroParisîech a tissé des liens privilégiés avec 'Afeid, Association française del' eau, de l'irrigation et du drainage Ihttp://afeid.montpellier.cemagref.fr/l. AgroParisTech bénéficie ainsi d'une veille, tant sur les préoccupations des professionnels et des experts que sur les changements du métier en termes d'évolution de compétences, tant pour la formation initiale que continue. D'autres formations de mastère abordent les relations eau-agriculture; citons notamment Politiques publiques et stratégies pour l'environnement IPPSE] et ngénierie et gestion de l'environnement IIGE].

\section{Autres formations continues}

La formation continue offre d'autres enseignements de plus courte durée pour des ingénieurs et administratifs en poste en France. Est notamment proposé dans ce cadre un parcours Pollutions diffuses d'origine agricole où les apprenants suivent une session sur les Fondamentaux de l'agronomie, puis quatre sessions de trois jours intégrant des aspects tech- 
niques et réglementaires de la gestion de l'eau. Une formation courte sur Les enjeux eau du Grenelle de l'environnement aborde le plan Écophyto 2018 et les enjeux liés aux aires d'alimentation de captages.

\section{Conclusion}

La thématique eau-agriculture est présente à AgroParisTech avec des adaptations aux différents cycles de formation lingénieur, master, mastère, doctorat). En formation ingénieur, la première année vise à donner des cadres de compréhension des grandes questions (qualité/quantité des eaux) et des modes d'approche possibles (approche biophysique/approche socio-économique). La deuxième année permet plus d'approfondir les thématiques eau (dans le domaine Environnement) et agriculture Idans le domaine Production) que de travailler sur les interfaces, sauf dans certains modules optionnels (projet de 2e année). La troisième année est le lieu de mise en situation professionnelle encadrée où l'on retrouve plus directement ces interfaces, que ce soit au travers d'exercices pédagogiques (jeu de rôle) ou de réponses à des commanditaires publics et privés, réalisés en petits groupes d'étudiants (projets dïngénieursl ou de manière autonome (stages de fin d'étude). La formation de master prépare préférentiellement à la recherche avec le plus souvent une orientation disciplinaire marquée, mais aussi des réflexions méthodologiques et appliquées sur l'interdisciplinarité nécessaire pour le traitement des questions eauagriculture dans toute leur complexité. La formation post-master et la formation continue, quant à elles, ciblent des publics bien différenciés. D'un côté on a des professionnels et des jeunes diplômés de niveau bac +5 généralement de formation technique qui souhaitent évoluer vers des fonctions de management et qui s'orientent vers des formations longues de type mastères spécialisés avec souvent une ouverture forte à l'international. De l'autre, des formations plus courtes permettent de répondre aux besoins d'ingénieurs et techniciens, voire chercheurs qui souhaitent actualiser les outils et méthodes mis en œuvre au quotidien. Nombre des formations présentées dans cet article n'existaient pas sous leur forme actuelle trois ou quatre ans auparavant. Ces changements, qui devraient se poursuivre à l'avenir. traduisent le besoin de s'adapter à une société qui évolue. Par ailleurs cette évolution se fait en lien étroit avec les unités de recherches associées à l'établissement et en collaboration avec d'autres établissements d'enseignement supérieur. que ce soit à Paris ou sur des sites régionaux comme Montpellier

Remerciements: les auteurs remercient les collègues qui ont permis d'améliorer ce texte par leurs remarques: Laure Vieuble, Ambroise de Montbel, Sophie Richard, Gilian Cadic, Erwan Personne, Thierry Rieu, Pierre-Yves Vion. 